Pacific Journal of Mathematics

A NECESSARY CONDITION ON THE EXTREME POINTS OF A
CLASS OF HOLOMORPHIC FUNCTIONS 


\title{
A NECESSARY CONDITION ON THE EXTREME POINTS OF A CLASS OF HOLOMORPHIC FUNCTIONS
}

\author{
FranK FORELLI
}

\begin{abstract}
We prove that if $f$ is an extreme point of the class $N(G, p)$ of all holomorphic functions $g$ on the region $G$ in $C^{n}$ such that $\operatorname{Re} g>0$ on $G$ and $g(p)=1$, and if three conditions on $G$ hold, then the Cayley transform $(f-1) /(f+1)$ is irreducible.
\end{abstract}

1. Introduction.

1.1. Let $G$ be a nonempty open connected subset of $C^{n}$. We will denote by $H(G)$ the class of all holomorphic functions on $G$, we will denote by $N(G)$ the class of all $f$ in $H(G)$ such that $\operatorname{Re} f>0$, and we will denote by $W(G)$ the class of all $f$ in $H(G)$ such that $|f| \leqq 1$. Thus $W(G)$ is the closed unit ball of the Banach algebra

$$
\{f: f \in H(G),\|f\|<\infty\}
$$

where

$$
\|f\|=\sup \{|f(z)|: z \in G\}
$$

Let $p \in G$ and let

$$
N(G, p)=\{f: f \in N(G), f(p)=1\} .
$$

Thus $N(G, p)$ is convex (and compact with respect to the compact open topology). Furthermore let

$$
W(G, p)=\{g: g \in W(G), g(p)=0\} \text {. }
$$

Thus

$$
N(G, p)=\{(1+g) /(1-g): g \in W(G, p)\} .
$$

We will denote (as is usual) by $\operatorname{Aut}(G)$ the group of holomorphic homeomorphisms of $G$, and by $H^{1}(G, Z)$ the first Cech cohomology group of $G$ with integer coefficients. We recall $[1, p .769]$ that if $g \in W(G)$, if $g$ is not constant, and if $g$ is not the product of two nonconstant members of $W(G)$, then $g$ is said to be irreducible. The purpose of this paper is to state and prove the following theorem.

THeORem 1.2. Let $G$ be such that (a) Aut $(G)$ acts transitively on $G$, and (b) $H^{1}(G, Z)=0$. Furthermore let $g \in W(G, p)$ and let $f=(1+g) /(1-g)$. If $N(G, p) \neq\{1\}$ and if $f$ is an extreme point of $N(G, p)$, then $g$ is irreducible. 
1.3. With regard to Theorem $\mathbf{1 . 2}$ if

$$
G=\left\{z: z \in C^{n},|z|<1\right\}
$$

and if $n \geqq 2$, then the fact that $g$ is irreducible does not imply that $f$ is extreme. For example if $g(z)=z_{1}$, then (as is easily seen) $g$ is irreducible. Whereas if $h(z)=z_{2}^{2} / 2$, then $g+e^{i \theta} h \in W(G, 0)$ and

$$
f=\frac{1}{2 \pi} \int_{0}^{2 \pi}\left[\left(1+g+e^{i \theta} h\right) /\left(1-g-e^{i \theta} h\right)\right] d \theta .
$$

If

$$
G=\{z: z \in C,|z|<1\},
$$

then (as is well known) $f$ is extreme if and only if $g$ is irreducible.

We do not know if Theorem 1.2 holds if we omit one or both of the hypotheses (a) and (b). With regard to this we remark that these hypotheses are not used in $\S \S 2$ and 3 . They are used only in $\S 4$.

2. The beginning of the proof of Theorem 1.2.

Proposition 2.1. If $N(G, p) \neq\{1\}$, then 1 is not an extreme point of $N(G, p)$.

Proof. We have $W(G, p) \neq\{0\}$.

Furthermore if $g \in W(G, p)$, then

$$
\operatorname{Re}(1+g)=1+\operatorname{Re} g>0,
$$

hence $1+g \in N(G, p)$. Likewise $1-g \in N(G, p)$, hence 1 is not an extreme point of $N(G, p)$.

Lemma 2.2. Let $g \in W(G, p)$ and let $f=(1+g) /(1-g)$. If $g=h^{2}$ where $h \in H(G)$ and if $N(G, p) \neq\{1\}$, then $f$ is not an extreme point of $N(G, p)$.

Proof. If $j=2 h /(1-g)$, then $f+j=(1+h) /(1-h)$ and $f-j=$ $(1-h) /(1+h)$, hence $f+j, f-j \in N(G, p)$. Thus if $h \neq 0$, then $f$ is not an extreme point of $N(G, p)$. If $h=0$, then by Proposition $2.1 f$ is not an extreme point of $N(G, p)$.

2.3. We recall the following theorem of Ahern and Rudin (which is proved by means of the theory of normal families) [1, Lemma 3.3 and the postscript on p. 777].

Proposition 2.4. If $g \in W(G, p)$ and if $g \neq 0$, then $g=a b$ where 
$a \in W(G, p), a$ is irreducible, and $b \in W(G)$.

2.5. We will denote (as is usual) by $\boldsymbol{D}$ the class of all $z$ in $\boldsymbol{C}$ such that $|z|<1$, by $\bar{D}$ the class of all $z$ in $C$ such that $|z| \leqq 1$, and by $T$ the class of all $z$ in $C$ such that $|z|=1$.

Proposition 2.6. If $z, w \in \overline{\boldsymbol{D}}$, then

$$
\left|z w+\frac{z+w}{2}\right| \leqq\left|1+\frac{z+w}{2}\right|
$$

Proof. Let $2<t<\infty$. If we define $f: \overline{\boldsymbol{D}} \times \overline{\boldsymbol{D}} \rightarrow \boldsymbol{C}$ by

$$
f(z, w)=\left(z w+\frac{z+w}{t}\right) /\left(1+\frac{z+w}{t}\right),
$$

then $|f|=1$ on $\boldsymbol{T} \times \boldsymbol{T}$, hence by the principle of maximum $|f| \leqq 1$ which will suffice to prove Proposition 2.6.

2.7. We recall that if $z, w \in C$ and if $z \neq 1$, then

$$
\operatorname{Re}[(1+z+2 w) /(1-z)]=\left(|1+w|^{2}-|z+w|^{2}\right) /|1-z|^{2} .
$$

Lemma 2.8. Let $g \in W(G, p)$ and let $f=(1+g) /(1-g)$. If $N(G, p) \neq\{1\}$ and if $f$ is an extreme point of $N(G, p)$, then $g=$ $a(s-a) /(1-\bar{s} a)$ where $a \in W(G, p), a$ is irreducible, $s \in C, s+\bar{s}=0$, and $0<|s| \leqq 1$.

Proof. By Proposition $2.1 g \neq 0$. Let $g=a b$ where $a \in W(G, p)$ and $b \in W(G)$.

Let $\lambda$ in $T$ be such that $\operatorname{Re}[\bar{\lambda} b(p)]=0$, let $c=\lambda a$, and let $d=$ $\bar{\lambda} b$. Thus $g=c d$. Let $s=d(p)$. Thus $s+\bar{s}=0$ and $|s| \leqq 1$. If $h=[(c+d) /(1-g)]-s$, then $h(p)=0$. Furthermore

$$
\operatorname{Re}(f+h)=\operatorname{Re}(f+h+s)=\operatorname{Re}[(1+g+c+d) /(1-g)],
$$

hence by (2.1)

$$
\operatorname{Re}(f+h)=\left(\left|1+\frac{c+d}{2}\right|^{2}-\left|c d+\frac{c+d}{2}\right|^{2}\right) /|1-g|^{2},
$$

hence by Proposition 2.6 $f+h \in N(G, p)$. Likewise $f-h \in N(G, p)$, thus $h=0$. We have

$$
c+d=s(1-g)=s(1-c d),
$$

hence

$$
d=(s-c) /(1+s c)=(s-c) /(1-\bar{s} c) .
$$


If $s=0$, then $d=-c$, hence $g=(i c)^{2}$. Thus by Lemma $2.2 s \neq 0$. Lemma 2.8 now follows from Proposition 2.4.

3. The action of $\operatorname{Aut}(G)$ on $N(G, p)$.

3.1. We define $\alpha$ : Aut $(G) \times N(G) \rightarrow N(G, p)$ by

$$
\alpha(Z, f)=(f \circ Z-\lambda) / \mu
$$

where $\lambda=i \operatorname{Im} f(Z(p))$ and $\mu=\operatorname{Re} f(Z(p))$. We will omit the proof (which is straightforward) of the following proposition.

Proposition 3.2. If $Y, Z \in \operatorname{Aut}(G)$, if $f, g \in N(G)$, and if $t>0$, then

$$
\alpha(Z, f+g)=\alpha \alpha(Z, f)+b \alpha(Z, g)
$$

where $a, b>0$ and $a+b=1$,

$$
\alpha(Z, t f)=\alpha(Z, f)
$$

and

$$
\alpha(Y Z, f)=\alpha(Z, \alpha(Y, f)) .
$$

Furthermore if $h \in N(G, p)$, then

$$
\alpha(I, h)=h .
$$

Proposition 3.3. Let $f \in N(G, p)$ and let $Z \in$ Aut $(G)$. If $f$ is an extreme point of $N(G, p)$, then $\alpha(Z, f)$ is an extreme point of $N(G, p)$.

Proof. Let $Y=Z^{-1}$. If $\alpha(Z, f)=a g+b h$ where $a, b>0$ and $g, h \in N(G, p)$, then by (3.1) and (3.2)

$$
\alpha(Y, \alpha(Z, f))=c \alpha(Y, g)+d \alpha(Y, h)
$$

where $c, d>0$ and $c+d=1$. Furthermore by (3.3) and (3.4)

$$
\alpha(Y, \alpha(Z, f))=f,
$$

hence

$$
f=c \alpha(Y, g)+d \alpha(Y, h) .
$$

Thus $f=\alpha(Y, g)$, hence

$$
\alpha(Z, f)=\alpha(Z, \alpha(Y, g))=g
$$

which completes the proof of Proposition 3.3. 
3.4. Although a paraphrase of Proposition 3.3 is proved in [2], it seemed worthwhile to give a proof here.

We define $\beta$ : Aut $(G) \times W(G, p) \rightarrow W(G, p)$ by

$$
\beta(Z, g)=\frac{1-\bar{\mu}}{1-\mu} \frac{g \circ Z-\mu}{1-\bar{\mu} g \circ Z}
$$

where $\mu=g(Z(p))$. We will omit the proof (which is straightforward) of the following proposition.

Proposition 3.5. Let $g \in W(G, p)$ and let $f=(1+g) /(1-g) . \quad$ If $Z \in \operatorname{Aut}(G)$, then

$$
\alpha(Z, f)=[1+\beta(Z, g)] /[1-\beta(Z, g)] .
$$

4. The end of the proof of Theorem 1.2.

Proposition 4.1. Let $a \in W(G)$. If $a$ is irreducible and if $H^{1}(G, Z)=0$, then $a(G)=\boldsymbol{D}$.

Proof. If $t \in \boldsymbol{D}-a(G)$, then since $H^{1}(G, Z)=0,(a-t) /(1-\bar{t} \alpha)=b^{2}$ where $b \in W(G)$. We have $a=\left(b^{2}+t\right) /\left(1+\bar{t} b^{2}\right)$. Thus if $t=-s^{2}$, then

$$
a=\frac{b-s}{1-\bar{s} b} \frac{b+s}{1+\bar{s} b}
$$

Since $a$ is irreducible either $(b-s) /(1-\bar{s} b) \in C$ or $(b+s) /(1+\bar{s} b) \in C$, hence $b \in C$, hence $a \in C$ which contradicts the fact that $a$ is irreducible.

Proposition 4.2. Let $g \in W(G, p)$ and let $f=(1+g) /(1-g)$. If $N(G, p) \neq\{1\}$, if $f$ is an extreme point of $N(G, p)$, and if $H^{1}(G, Z)=0$, then $g(G)=D$.

Proof. By Lemma 2.8

$$
g=a(s-a) /(1-\bar{s} a)
$$

where $a \in W(G, p), a$ is irreducible, and $s \in \overline{\boldsymbol{D}}$. By Proposition 4.1 $a(G)=D$, hence by (4.1) $g(G)=D$.

4.3. We will now prove Theorem 1.2. By Lemma 2.8

$$
g=a(s-a) /(1-\bar{s} a)
$$

where $a \in W(G, p), a$ is irreducible, $s \in \overline{\boldsymbol{D}}$, and $s \neq 0$.

The roots of $\bar{s} x^{2}+2 i x-s$ are $-i\left[1 \pm(1-s \bar{s})^{1 / 2}\right] / \bar{s}$. Thus if $\lambda=-i\left[1-(1-s \bar{s})^{1 / 2}\right] / \bar{s}$, then 


$$
\bar{s} \lambda^{2}+2 i \lambda-s=0 .
$$

Furthermore if $|s|<1$, then $|\lambda|<1$. Let $\mu=-\lambda^{2}$, let $z$ in $G$ be such that $g(z)=\mu$ (Proposition 4.2), and let $Z$ in Aut $(G)$ be such that $Z(p)=z$. Thus $g(Z(p))=\mu$. If $b=a \circ Z$, then by (4.2)

$$
\begin{aligned}
(g \circ Z-\mu) /(1-\bar{\mu} g \circ Z) & =\left(b \frac{s-b}{1-\bar{s} b}+\lambda^{2}\right) /\left(1+\bar{\lambda}^{2} b \frac{s-b}{1-\bar{s} b}\right) \\
& =\left[\lambda^{2}+\left(s-\bar{s} \lambda^{2}\right) b-b^{2}\right] /\left[1-\left(\bar{s}-s \bar{\lambda}^{2}\right) b-\bar{\lambda}^{2} b^{2}\right] .
\end{aligned}
$$

Furthermore by (4.3) $s-\bar{s} \lambda^{2}=2 i \lambda$, hence

$$
\begin{aligned}
(g \circ Z-\mu) /(1-\bar{\mu} g \circ Z) & =\left(\lambda^{2}+2 i \lambda b-b^{2}\right) /\left(1+2 i \bar{\lambda} b-\bar{\lambda}^{2} b^{2}\right) \\
& =[(\lambda+i b) /(1+i \bar{\lambda} b)]^{2} .
\end{aligned}
$$

Thus

$$
\beta(Z, g)=\left(\frac{1-\bar{\mu}}{1-\mu}\right)\left(\frac{\lambda+i b}{1+i \bar{\lambda} b}\right)^{2}=h^{2}
$$

where $h \in W(G, p)$. Thus by Lemma 2.2 and Proposition $3.5 \alpha(Z, f)$ is not an extreme point of $N(G, p)$ which contradicts Proposition 3.3. Thus $|s|=1$.

We have

$$
g=a s(1-\bar{s} \alpha) /(1-\bar{s} \alpha)=s a
$$

which completes the proof of Theorem 1.2.

\section{REFERENCES}

1. P. Ahern and W. Rudin, Factorizations of bounded holomorphic functions, Duke Math. J., 39 (1972), 767-777.

2. F. Forelli, Measures whose Poisson integrals are pluriharmonic II, Illinois J. Math., 19 (1975), 584-592.

Received August 2, 1976 and in revised form June 23, 1977. Partially supported by the National Science Foundation.

The University of Wisconsin-Madison

MADISON, WI 53706 


\section{PACIFIC JOURNAL OF MATHEMATICS}

EDITORS

RICHARD ARENS (Managing Editor)

University of California

Los Angeles, California 90024

C. W. Curtis

University of Oregon

Eugene, OR 97403

C. C. MOORE

J. DUGUNDJI

Department of Mathematics University of Southern Californa Los Angeles, California 90007

R. Finn AND J. Milgram Stanford University Stanford, California 94305

University of California

Berkeley, CA 94720

\section{ASSOCIATE EDITORS}

E. F. BeCKENBACH

B. H. NeUMANN

F. WOLF

K. YosHida

\section{SUPPORTING INSTITUTIONS}

UNIVERSITY OF BRITISH COLUMBIA UNIVERSITY OF SOUTHERN CALIFORNIA CALIFORNIA INSTITUTE OF TECHNOLOGY UNIVERSITY OF CALIFORNIA MONTANA STATE UNIVERSITY UNIVERSITY OF NEVADA, RENO STANFORD UNIVERSITY UNIVERSITY OF TOKYO UNIVERSITY OF UTAH NEW MEXICO STATE UNIVERSITY WASHINGTON STATE UNIVERSITY OREGON STATE UNIVERSITY UNIVERSITY OF WASHINGTON UNIVERSITY OF OREGON OSAKA UNIVERSITY 


\section{Pacific Journal of Mathematics \\ Vol. 73, No. 1 \\ March, 1977}

Thomas Robert Berger, Hall-Higman type theorems. $V \ldots \ldots \ldots \ldots \ldots \ldots \ldots$

Frank Peter Anthony Cass and Billy E. Rhoades, Mercerian theorems via

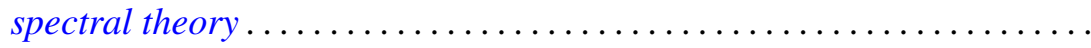

Morris Leroy Eaton and Michael David Perlman, Generating $\mathrm{O}(n)$ with

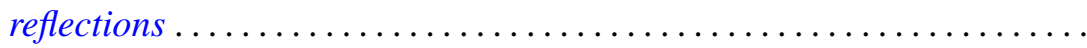

Frank John Forelli, Jr., A necessary condition on the extreme points of a

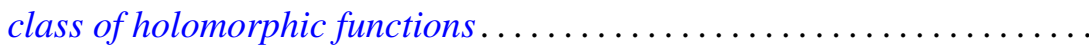

Melvin F. Janowitz, Complemented congruences on complemented

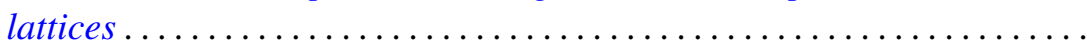

Maria M. Klawe, Semidirect product of semigroups in relation to amenability, cancellation properties, and strong $F \phi$ lner conditions....

Theodore Willis Laetsch, Normal cones, barrier cones, and the "spherical image" of convex surfaces in locally convex spaces ................

Chao-Chu Liang, Involutions fixing codimension two knots.............

Joyce Longman, On generalizations of alternative algebras .............

Giancarlo Mauceri, Square integrable representations and the Fourier

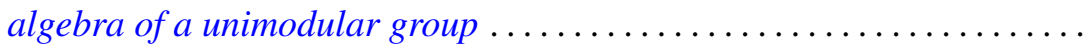

J. Marshall Osborn, Lie algebras with descending chain condition...

John Robert Quine, Jr., Tangent winding numbers and branched

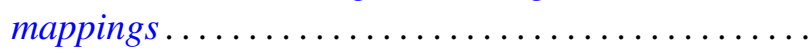

Louis Jackson Ratliff, Jr. and David Eugene Rush, Notes on ideal covers and associated primes .

H. B. Reiter and N. Stavrakas, On the compactness of the hyperspace of faces.

Walter Roth, A general Rudin-Carlson theorem in Banach-spaces ..

Mark Andrew Smith, Products of Banach spaces that are uniformly rotund in every direction.

Roger R. Smith, The R-Borel structure on a Choquet simplex ...

Gerald Stoller, The convergence-preserving rearrangements of real infinite series. ...

Graham H. Toomer, Generalized homotopy excision theorems modulo a Serre class of nilpotent groups...

Norris Freeman Weaver, Dehn's construction and the Poincaré conjecture....

Steven Howard Weintraub, Topological realization of equivariant intersection forms... 